\title{
The Group Theoretical Aspects of Infinitesimal Riemann-Hilbert Transform and Hidden Symmetry
}

\author{
Wu Yong-Shi $\star$, $\star \star$ \\ Centre de Physique Theorique, C.N.R.S.-Luminy, Case 907, F-13288 Marseille Cedex 9, France \\ and Max Planck Institut für Physik and Astrophysik, D-8000 München 40, Federal Republic of \\ Germany
}

\begin{abstract}
We obtain explicit expressions for infinitesimal regular RiemannHilbert $(\mathrm{RH})$ transforms. Using them, the group theoretical aspects of infinitesimal RH transforms are discussed with an eye to the comparison with the hidden symmetry transformations proposed by us before. We find that the RH transforms have very rich group structure; e.g. in the 2-d principal chiral models, their group contains two Kac-Moody algebras as subalgebras. But not all of them are nontrivial hidden symmetries of the theory.
\end{abstract}

\section{Introduction}

Very recently there has been much progress in understanding the existence and Liealgebraic structure of an infinite-parameter hidden symmetry in 2-d principal chiral models [1-7] and 4-d self-dual Yang-Mills equations [8-12]. Basically, there are two approaches to this problem. One is explicit construction of the infinitesimal hidden symmetry transformations for the basic fields under consideration $[1-5,7]$ and $[9,11,12]$. Another one is to use the regular infinitesimal Riemann-Hilbert (RH) transform for the auxiliary quantities - the solutions to the linearization equations [6 and 10]. The original motivation of this paper was to investigate the connection between these two approaches.

However, during the course of this study, it gradually became clear that our explicit expressions for infinitesimal RH transforms and the evaluation of their commutators are in fact generally true. Therefore the study has developed into a general discussion of the group theoretical aspects of (infinitesimal) RH transforms which have been already a powerful tool for solving many nonlinear equations. But we prefer to start with a concrete example, in order to make the presentation not too abstract at the beginning.

* Institute for Advanced Study, Princeton, NJ 08540, USA

$\star \star$ Permanent address: Institute of Theoretical Physics, Academia Sinica, Box 2735, Beijing, People's Republic of China 


\section{Brief Review of RH Transforms}

First let us consider 2-d principal chiral models as an example. The equations of motion are

$$
\partial_{\xi}\left(g^{-1} \partial_{\eta} g\right)+\partial_{\eta}\left(g^{-1} \partial_{\xi} g\right)=0,
$$

where $\xi$ and $\eta$ are light-cone variables and the basic fields $g(\xi, \eta)$ takes values in a matrix group $G$. The Riemann-Hilbert transform method [13,14] starts with the linearization system for the theory

$$
\partial_{\xi} U=\frac{\ell}{1-\ell} A_{\xi} U, \quad \partial_{\eta} U=\frac{-\ell}{1+\ell} A_{\eta} U,
$$

where $\ell$ is a complex parameter and

$$
A_{\xi}=g^{-1} \partial_{\xi} g, \quad A_{\eta}=g^{-1} \partial_{\eta} g .
$$

Note that $A_{\xi}$ and $A_{\eta}$ are independent of $\ell$, but the solution $U$ to the system (2) is a function of both $\ell$ and $\xi, \eta$.

We can always normalize $U$, so that

$$
U=(\ell=0 ; \xi, \eta)=1 .
$$

The key point here is that the necessary and sufficient conditions for the existence of a solution $U(\ell ; \xi, \eta)$ to the system (2) are just the equations of motion (1) and the following curvature-free condition derivable from the definition (3)

$$
\partial_{\xi} A_{\eta}-\partial_{\eta} A_{\xi}+\left[A_{\xi}, A_{\eta}\right]=0 .
$$

Therefore, from a solution to the system (2) we can reconstruct

$$
A_{\xi}=\partial_{\xi} \dot{U}(\ell=0), \quad A_{\eta}=-\partial_{\eta} \dot{U}(\ell=0)
$$

(where a dot denotes differentiation with respect to $\ell$ ), and then, according to Eq. (3), obtain $g(\xi, \eta)$ by integration.

The basic idea of the RH transform method for solving Eq. (1) is first to generate a new solution $U^{\prime}$ of the linearization system (2) from an old $U$ by means of the $\mathrm{RH}$ transform and then obtain a new solution $g^{\prime}$ of Eq. (2) from $U^{\prime}$ in the above way. The RH transform from $U$ to $U^{\prime}$ consists of the following steps [13]:

1) To select, in the complex $\ell$-plane, a small circle $C$ with the centre at the origin such that $U(\ell)$ is analytic on $C_{+} \cup C$ (hereafter $C_{ \pm}$denotes the inside and outside of $C$ respectively).

2) To choose a matrix function of only $\ell, u(\ell) \in G$, which is analytic on $C$ and forms the kernal

$$
K(\ell)=U(\ell) \cdot u(\ell) \cdot U(\ell)^{-1}, \quad(\ell \in C) .
$$

3) To consider the following RH problem of finding a pair of functions $X_{ \pm}(\ell)$ which are analytic in $C_{ \pm}$and continuous on $C$, respectively, such that

$$
X_{-}(\ell)=X_{+}(\ell) K(\ell)
$$

(We can always normalize $X_{ \pm}(\ell)$ so that

$$
\left.X_{+}(\ell=0)=1\right) \text {. }
$$


4) To introduce a new $U^{\prime}$ by

$$
U^{\prime}(\ell)= \begin{cases}X_{+}(\ell) U(\ell) & \text { in } C_{+} \\ X_{-}(\ell) U(\ell) u(\ell)^{-1} & \text { in } C_{-}\end{cases}
$$

It can be shown [6] that this $U^{\prime}(\ell)$ is also a solution to the linearization system (2) with the new potential

$$
A_{\xi}^{\prime}=A_{\xi}+\partial_{\xi} \dot{X}_{+}(0), \quad A_{\eta}^{\prime}=A_{\eta}-\partial_{\eta} \dot{X}_{-}(0) .
$$

(We will be restricted to the case in which $\operatorname{det} X_{ \pm}(\ell) \neq 0$ for all $\ell$, the so-called regular $\mathrm{RH}$ transform.)

The transform $U(\ell) \mapsto U^{\prime}(\ell)$ can be written as an integral equation. To derive it, note that as $X_{-}(\ell)$ is analytic in $C_{-} \cup C$ (including $\ell=\infty$ ), we have

$$
\frac{1}{2 \pi i} \int_{c} d \ell^{\prime} \frac{X_{-}\left(\ell^{\prime}\right)}{\ell^{\prime}\left(\ell^{\prime}-\ell\right)}=0 .
$$

Using Eq. (7) and

$$
\int_{c} d \ell^{\prime} \frac{1}{\ell^{\prime}\left(\ell^{\prime}-\ell\right)}=0, \quad\left(\ell \in C_{+}\right)
$$

and noting that according to Eq. (8), $\left[X_{+}(\ell)-1\right] / \ell$ is analytic in $C_{+} \cup C$, we obtain

$$
X_{+}(\ell)-1+\frac{1}{2 \pi i} \int_{c} d \ell^{\prime} \frac{X_{+}\left(\ell^{\prime}\right)\left[K\left(\ell^{\prime}\right)-1\right]}{\ell^{\prime}\left(\ell^{\prime}-\ell\right)}=0, \quad\left(\ell \in C_{+}\right) .
$$

Multiplying $U(\ell)$ from the right and using Eqs. (6) and (9), it follows that for $\ell \in C_{+}$

$$
U^{\prime}(\ell)+\frac{1}{2 \pi i} \int_{c} d \ell^{\prime} \frac{\ell}{\ell^{\prime}\left(\ell^{\prime}-\ell\right)} U^{\prime}\left(\ell^{\prime}\right)\left[u\left(\ell^{\prime}\right)-1\right] U\left(\ell^{\prime}\right)^{-1} U(\ell)=U(\ell) .
$$

\section{Explicit Expressions for Infinitesimal RH Transforms}

For infinitesimal RH transforms we are able to obtain explicit solutions to the integral equation (14). For in this case we have

where

$$
\delta U(\ell) \equiv U^{\prime}(\ell)-U(\ell)=\Delta(\ell) \cdot U(\ell), \quad\left(\text { for } \quad \ell \in C_{+}\right),
$$

$$
\Delta(\ell)=\frac{-1}{2 \pi i} \int_{\mathrm{c}} d \ell^{\prime} \frac{\ell}{\ell^{\prime}\left(\ell^{\prime}-\ell\right)} U\left(\ell^{\prime}\right) v\left(\ell^{\prime}\right) U\left(\ell^{\prime}\right)^{-1},
$$

and $v\left(\ell^{\prime}\right)=u\left(\ell^{\prime}\right)-1$ is an infinitesimal function in the Lie algebra of $G$. The infinitesimal variation of $A_{\mu}$ is given by

$$
\delta A_{\xi}=\partial_{\xi} \delta \dot{U}(0), \quad A_{\eta}=-\partial_{\eta} \delta \dot{U}(0)
$$

To be more explicit, we assume

$$
v(\ell)=\alpha^{a} T_{a} \ell^{-k},
$$

where $T_{a}$ are the generators of the Lie algebra of $G, \alpha^{a}$ are infinitesimal constants and $k$ is an integer (because $v(\ell)$ was required to be analytic only on $C, k$ can be positive, 
negative or zero). The corresponding $\delta U(\ell)$ will be denoted as $\delta_{\alpha}^{(k)} U^{1}$. Note that for $\ell \in C_{+}$and $\ell^{\prime} \in C$ we always have $|\ell| \ell^{\prime} \mid<1$, so we have the expansion

$$
\frac{1}{\ell^{\prime}\left(\ell^{\prime}-\ell\right)}=\sum_{n=0}^{\infty} \frac{\ell^{n+1}}{\ell^{\prime n+2}} \text {. }
$$

Because $U(\ell)$ is analytic on $C_{+} \cup C$, we can have the expansion

$$
U(\ell ; x) T_{a} U(\ell ; x)^{-1}=\sum_{m=0}^{\infty} \ell^{m} T_{a}^{(m)}(x) \quad\left(\ell \in C_{+} \cup C\right)
$$

(where $x=(\xi, \eta)$ ). Upon substitution of Eqs. (17) and (18) into Eq. (16) and by using Eq. (19), and

$$
\frac{1}{2 \pi i} \int_{c} d \ell^{\prime} \ell^{\prime-k}=\delta_{k, 1}, \quad(k \in \mathbb{Z})
$$

it follows from Eq. (15)

$$
\delta_{\alpha}^{(k)} U(\ell ; x)=-\alpha^{a} \sum_{n=0}^{\infty} \ell^{n+1} T_{a}^{(n+k+1)}(x) U(\ell ; x) .
$$

To examine the implications of Eq. (21) for different values of $k$, we observe that as $U\left(\ell^{\prime}\right)$ is analytic around $\ell^{\prime}=0$ and $U\left(\ell^{\prime}=0\right)=1$, in Eq. (19) we should have

$$
T_{a}^{(m=0)}(x)=T_{a}, \quad T_{a}^{(m<0)}(x) \equiv 0 .
$$

Let us consider the following three cases respectively:

1) $k=0$ : Eqs. (21) and (19) give

$$
\delta_{\alpha}^{(0)} U(\ell)=-\alpha^{a}\left[U(\ell), T_{a}\right]
$$

The corresponding $\delta_{\alpha}^{(0)} A_{\mu}$ can be obtained from Eq. (16); e.g.,

$$
\delta_{\alpha}^{(0)} A_{\xi}=-\alpha^{a}\left[A_{\xi}, T_{a}\right]
$$

2) $k<0$ : using Eq. (22) and noting $|k| \geqq 1$,

$$
\begin{aligned}
\delta_{\alpha}^{(k)} U(\ell) & =U(\ell)\left(-\alpha^{a} T_{a} \ell^{|k|}\right), \\
\delta_{\alpha}^{(k)} A_{\xi} & =\delta_{\alpha}^{(k)} A_{\eta}=0 .
\end{aligned}
$$

The result $\left(24^{\prime}\right)$ is not surprising, because in the original linearization system $(2), U(\ell)$ is determined only up to a right-multiplicative matrix which is independent of $(\xi, \eta)$ but may depend on $\ell$. So Eq. (24) implies that all the infinitesimal RH transforms with $k \leqq-1$ are trivial in the sense that they do not give new solutions $g^{\prime}(\xi, \eta)$ to the equations of motion (1), although formally they give global right-multiplication transforms for $U(\ell)$.

3) $k>0:$ in this case,

$$
\delta_{\alpha}^{(k)} U(\ell)=-\alpha^{a} \sum_{n=0}^{\infty} \ell^{n+1} T_{a}^{(n+1+k)}(x) \cdot U(\ell)
$$

1 The generic infinitesimal (regular) RH transform is a linear combination of $\delta_{\alpha}^{(k)} U$ 


$$
\begin{aligned}
& =-U(\ell)\left(\alpha^{a} T_{a} \ell^{-k}\right)+\sum_{n=0}^{k} \alpha^{a} \ell^{n-k} T_{a}^{(n)}(x) \cdot U(\ell), \\
\delta_{\alpha}^{(k)} A_{\xi} & =-\alpha^{a} \partial_{\xi} T_{a}^{(k+1)}(x) .
\end{aligned}
$$

Thus, excluding the trivial transformations (24) we obtain from Eqs. (23) and (25) an infinite set of transformations (indexed with zero and positive integers) all of which generate nontrivial solutions to the equations of motion (1).

To compare the above results with those in our approach $[2,4]$, we encapsulate the transformations (23) and (25) as follows. Introducing another complex parameter $\ell^{\prime}$ we can consider the sum

$$
\delta_{\alpha}^{\prime} U(\ell)=\sum_{k=0}^{\infty} \ell^{\prime k} \delta_{\alpha}^{(k)} U(\ell)
$$

which can be viewed as a parametric (infinitesimal) transformation for $U(\ell)$. Correspondingly,

$$
\begin{aligned}
\delta_{\alpha}^{\prime} A_{\xi}=\sum_{k=0}^{\infty} \ell^{\prime k} \delta_{\alpha}^{(k)} A_{\xi} & =-\frac{1}{\ell^{\prime}} \partial_{\xi}\left\{U\left(\ell^{\prime}\right) T_{\alpha} U\left(\ell^{\prime}\right)^{-1}\right\} \\
& =-D_{\xi}\left\{U\left(\ell^{\prime}\right) T_{\alpha} U\left(\ell^{\prime}\right)^{-1}\right\}, \\
\delta_{\alpha}^{\prime} A_{\eta}=\frac{1}{\ell^{\prime}} \partial_{\eta}\left\{U\left(\ell^{\prime}\right) T_{\alpha} U\left(\ell^{\prime}\right)^{-1}\right\} & =-D_{\eta}\left\{U\left(\ell^{\prime}\right) T_{\alpha} U\left(\ell^{\prime}\right)^{-1}\right\},
\end{aligned}
$$

where $T_{\alpha} \equiv \alpha^{a} T_{a}, D_{\mu}=\partial_{\mu}+\left[A_{\mu}, \quad\right]$, and we have used Eqs. $\left(23^{\prime}\right),\left(25^{\prime}\right),(19)$ and, in particular for the last equalities, the system of equations (2) (with $\ell$ replaced by $\ell^{\prime}$ ) for $U\left(\ell^{\prime}\right)$. In general we have

$$
\delta A_{\mu}=\delta\left(g^{-1} \partial_{\mu} g\right)=D_{\mu}\left(g^{-1} \delta g\right) .
$$

Therefore, from Eqs. (27) and (27') we can read off the parametric (infinitesimal) transformation for the basic field $g(\xi, \eta)$ corresponding to Eq. (26) as follows:

$$
\delta_{\alpha}^{\prime} g=-g U\left(\ell^{\prime}\right) T_{\alpha} U\left(\ell^{\prime}\right)^{-1}
$$

It is none other than the infinitesimal hidden symmetry transformation proposed before in our explicit approach $[2,4]$. In some sense here we have found its origin from the infinitesimal RH transform. It can be easily verified that with Eqs. (23) and (25) substituted into it and by exchanging the order of two infinite sums, the definition (26) leads to the following formula

$$
\begin{aligned}
\delta_{\alpha}^{\prime} U(\ell) \cdot U(\ell)^{-1} & =-\alpha^{a} \sum_{k=0}^{\infty} \sum_{n=k+1}^{\infty} \ell^{\prime k} \ell^{n-k} T_{a}^{(n)}(x) \\
& =-\ell \alpha^{a} \sum_{n=1}^{\infty} \sum_{k=0}^{n-1} \ell^{\prime k} \ell^{n-k-1} T_{a}^{(n)}(x) \\
& =\frac{\ell}{\ell-\ell^{\prime}}\left\{U\left(\ell^{\prime}\right) T_{\alpha} U\left(\ell^{\prime}\right)^{-1}-U(\ell) T_{\alpha} U(\ell)^{-1}\right\}
\end{aligned}
$$

as we proved in [7].

Now that we already have the explicit expressions (23)-(25) for the action of 
infinitesimal RH transformations on $U(\ell)$, we can use them directly to check that these actions on $U(\ell)$ provide a representation of the Kac-Moody algebra (without the center) [15] $G \otimes C\left[\ell, \ell^{-1}\right]$, where $C\left[\ell, \ell^{-1}\right]$ is the algebra of Laurent polynomials in $\ell$ and $\ell^{-1}$; i.e., the following commutation relations hold for any integer $j$ and $k$ :

$$
\left[\delta_{a}^{(j)}, \delta_{b}^{(k)}\right] U=C_{a b}^{c} \delta_{c}^{(j+k)} U, \quad(j, k \in \mathbb{Z}),
$$

where $C_{a b}^{c}$ are structure constants of the Lie algebra of $G$. In fact, the proof for the case of both $j$ and $k<0$ is trivial. For the case of both $j$ and $k \geqq 0$, we can make use Eq. (30) to obtain a commutator for $\delta_{\alpha}^{\prime}$ and $\delta_{\beta}^{\prime \prime}$, and then expand it in powers of $\ell^{\prime}$ and $t^{\prime \prime}$. The case of $j<0$ and $k>0$ is easy to check too:

$$
\begin{aligned}
{\left[\delta_{a}^{(j)}, \delta_{b}^{(k)}\right] U=} & -\ell^{-j} \delta_{b}^{(k)} U T_{a}-\sum_{n=0}^{k} \ell^{n-k}\left\{T_{b}^{(n)}(x) \delta_{a}^{(j)} U+\delta_{a}^{(j)} T_{b}^{(n)}(x) U\right\} \\
& -\{(a, j) \leftrightarrow(b, k)\} .
\end{aligned}
$$

Using

$$
\delta_{a}^{(j)} T_{b}^{(n)}(x)=-C_{a b}^{c} T_{c}^{(n+j)}(x), \quad(j<0),
$$

which can be derived from the action of $\delta_{a}^{(j)}$ on Eq. (19), the commutator (31) for $j<0, k>0$ follows from Eq. (32). The advantage of our development here is the fact that to check the infinite-parameter Lie Algebra for RH transforms it is sufficient to use the explicit expressions for $\delta_{\alpha}^{(j)} U$. We avoided the introduction of certain cumbersome auxiliary quantities such as

$$
G\left(\ell^{\prime}, \ell\right) \equiv \sum_{m, n=0}^{\infty} G^{m, n} \ell^{\prime m} \ell^{n}=\frac{1}{\ell^{\prime}-\ell}\left\{\ell^{\prime}-\ell U\left(\ell^{\prime}\right)^{-1} U(\ell)\right\}
$$

which appeared in previous treatments (see, e.g., [6]). This fact makes our treatment greatly simplified both conceptually and operationally.

However, we would like to emphasize again that though formally the $\mathrm{RH}$ transforms (23)-(25) acting on $U(\ell)$ form the Kac-Moody algebra $G \otimes C\left[\ell, \ell^{-1}\right]$, not all the generators of it correspond to true and nontrivial symmetry of the theory. As we pointed out before, half the set of generators, i.e. $\delta_{\alpha}^{(j)} U$ with $j<0$, do not lead to nontrivial transformations for the basic field $g(x)$ : actually

$$
\delta_{\alpha}^{(j)} g(x)=0, \quad(j<0)
$$

(One may wonder if $\delta_{\alpha}^{(j)} g(j<0)$ could be some specific left global transformations dependent on $\ell$. In fact, if we act $\delta_{\beta}^{(j)}(j<0)$ on Eq. (29), it is easy to see that only Eq. (34) is consistent with

$$
\left.\left[\delta_{a}^{(j)}, \delta_{b}^{(k)}\right] g=C_{a b}^{c} \delta_{c}^{(j+k)} g, \quad(j<0, k>0) .\right)
$$

So in our opinion, for the infinitesimal RH transforms considered above (and also in [10]), only those with non-negative integer indices are true nontrivial symmetry transformations in the model, and they merely form the subalgebra $G \otimes C[\ell]$.

\section{Another Infinite Set of RH Transforms}

To extend the nontrivial hidden symmetry from $G \otimes C[\ell]$ to $G \otimes C\left[\ell, \ell^{-1}\right]$ (as we 
did in [7] in our previous explicit approach) in the $\mathrm{RH}$ transform approach, let us consider the alternative form of the linearization system

$$
\partial_{\xi} W=\frac{1}{\ell-1} A_{\xi} W, \quad \partial_{\eta} W=\frac{-1}{\ell+1} A_{\eta} W,
$$

with

$$
W(\ell=0)=g(x)^{-1} .
$$

From Eq. (36) it is easy to see that

$$
A_{\mu}=-\partial_{\mu} W(\ell=0) \cdot W(\ell=0)^{-1} .
$$

For Eq. (36) we can introduce RH transforms using exactly the same treatment given in Sect. II.

Suppose we already have a solution $W(\ell)$ to Eq. (36) which is analytic around $\ell=0$. Let us choose a small circle $\widetilde{C}$ in the complex $\ell$-plane, with center at the origin, such that $W(\ell)$ is analytic on $\widetilde{C}_{+} \cup \widetilde{C}$. Introduce a kernal, which is defined on $\widetilde{C}$, as follows:

$$
H(\ell)=W(\ell) \tilde{u}(\ell) W(\ell)^{-1}, \quad(\ell \in \widetilde{C}),
$$

where $\tilde{u}(\ell) \in G$ is analytic on $\widetilde{C}$. And then consider the $\mathrm{RH}$ problem associated with it:

$$
Y_{-}(\ell)=Y_{+}(\ell) H(\ell)
$$

where $Y_{ \pm}(\ell)$ are analytic in $\tilde{C}_{ \pm}$and continuous on $\tilde{C}$ respectively. We shall normalize $Y_{ \pm}(\ell)$ so that ${ }^{2}$

$$
Y_{-}(\ell=\infty)=1 \text {. }
$$

A new solution to Eq. (36) can be obtained by defining

$$
W^{\prime}(\ell)= \begin{cases}Y_{+}(\ell) W(\ell) & \text { in } C_{+} \\ Y_{-}(\ell) W(\ell) \tilde{u}(\ell)^{-1} & \text { in } C_{-} .\end{cases}
$$

The corresponding potential is given by

$$
A_{\mu}^{\prime}=Y_{+}(0) A_{\mu} Y_{+}(0)^{-1}+Y_{+}(0) \partial_{\mu} Y_{+}(0)^{-1}
$$

which, in turn, corresponds to the following solution-generating transformation for $g(x)$

$$
g(x) \mapsto g^{\prime}(x)=g(x) \cdot Y_{+}(0)^{-1} .
$$

From the Cauchy formulas for $Y_{ \pm}(\ell)$

$$
\begin{gathered}
\frac{1}{2 \pi i} \int_{\tilde{c}} d \ell^{\prime} \frac{Y_{-}\left(\ell^{\prime}\right)}{\ell^{\prime}-\ell}=1, \quad\left(\ell \in \tilde{C}_{+}\right), \\
\frac{1}{2 \pi i} \int_{\tilde{c}} d \ell^{\prime} \frac{Y_{+}\left(\ell^{\prime}\right)}{\ell^{\prime}-\ell}=Y_{+}(\ell), \quad\left(\ell \in \widetilde{C}_{+}\right),
\end{gathered}
$$

2 Note that in this case we cannot choose $Y_{+}(\ell=0)=1$ as we did in Sect. II; for this would lead to only the identity transformation for $A_{\mu}$, as is clear from Eqs. (42) and (38) 
it follows that

$$
Y_{+}(\ell)=1+\frac{1}{2 \pi i} \int_{\tilde{c}} d \ell^{\prime} \frac{Y_{+}\left(\ell^{\prime}\right)\left[1-H\left(\ell^{\prime}\right)\right]}{\ell^{\prime}-\ell}, \quad\left(\ell \in \tilde{C}_{+}\right) .
$$

For infinitesimal RH transforms we have

$$
\tilde{\delta} Y_{+}(\ell) \equiv Y_{+}(\ell)-1=\frac{-1}{2 \pi i} \int_{\tilde{c}} d \ell^{\prime} \frac{W\left(\ell^{\prime}\right) \tilde{v}\left(\ell^{\prime}\right) W\left(\ell^{\prime}\right)^{-1}}{\ell^{\prime}-\ell}
$$

and

$$
\tilde{\delta} W(\ell) \equiv W^{\prime}(\ell)-W(\ell)=\tilde{\delta} Y_{+}(\ell) \cdot W(\ell),
$$

where $\tilde{v}(\ell)=\tilde{u}(\ell)-1$ is an infinitesimal function taking values in the Lie algebra of $G$. Without losing generality we can assume

$$
\tilde{v}(\ell)=\alpha^{a} T_{a} \ell^{-k}, \quad(k \in \mathbb{Z}) .
$$

The corresponding $\mathrm{RH}$ transform is explicitly given by

$$
\tilde{\delta}_{\alpha}^{(k)} W(\ell)=-\alpha^{a} \sum_{n=0}^{\infty} \ell^{n} \widetilde{T}_{a}^{(n+k)}(x) W(\ell),
$$

where $\widetilde{T}_{a}^{(n)}(x)(n \geqq 0)$ are defined by the expansion

$$
W(\ell) T_{a} W(\ell)^{-1}=\sum_{n=0}^{\infty} \ell^{n} \tilde{T}_{a}^{(n)}(x), \quad\left(\ell \in \widetilde{C}_{+} \cup \widetilde{C}\right),
$$

and

$$
\tilde{T}_{a}^{(n)}(x) \equiv 0 \quad \text { for } \quad n<0 .
$$

Concretely we have to discuss the cases of $k \leqq 0$ and $k>0$ respectively:

1) For $k \leqq 0$,

$$
\tilde{\delta}_{\alpha}^{(k)} W(\ell)=W(\ell)\left\{-\alpha^{a} T_{a} \ell^{|k|}\right\}
$$

It corresponds to

$$
\tilde{\delta}_{\alpha}^{(k)}(g)=\left\{\begin{array}{cc}
g \alpha^{a} \widetilde{T}_{a}^{(0)}(x) & \text { for } k=0 \\
0 & \text { for } k<0,
\end{array}\right.
$$

which are trivial in the sense that they do not lead to a nontrivial transformation for $A_{\mu}$ :

2) For $k>0$,

$$
\delta_{\alpha}^{(k)} A_{\mu}=0 \quad \text { for } k \leqq 0
$$

$$
\tilde{\delta}_{\alpha}^{(k)} W(\ell)=-\ell^{k} \sum_{n=k}^{\infty} \ell^{n} \alpha^{a} \tilde{T}_{a}^{(n)}(x) \cdot W(\ell)
$$

According to Eq. (44), it gives rise to

$$
\tilde{\delta}_{\alpha}^{(k)} g=g\left[\alpha^{a} \tilde{T}_{a}^{(k)}(x)\right] \text { for } k>0 .
$$

If we use the following definition to summarize these transformations

$$
\tilde{\delta}_{\alpha}^{\prime} g=\sum_{k=0}^{\infty} \ell^{\prime k} \tilde{\delta}_{\alpha}^{(k)} g
$$


from Eq. (53) and (56) we find

$$
\delta_{\alpha}^{\prime} g=g W\left(\ell^{\prime}\right) T_{\alpha} W\left(\ell^{\prime}\right)^{-1},
$$

which is just what we proposed previously in [7] for the second half of the infinite set of hidden symmetry transformations giving rise to the Kac-Moody algebra $G \otimes C\left[\ell, \ell^{-1}\right]$. It can be also verified that ${ }^{3}$

$$
\tilde{\delta}_{\alpha}^{\prime} W(\ell) \equiv \sum_{k=1}^{\infty} \ell^{\prime k} \tilde{\delta}_{\alpha}^{(k)} W(\ell)
$$

satisfies the following relation

$$
\tilde{\delta}_{\alpha}^{\prime} W(\ell) \cdot W(\ell)^{-1}=\frac{\ell^{\prime}}{\ell-\ell^{\prime}}\left\{W\left(\ell^{\prime}\right) T_{\alpha} W\left(\ell^{\prime}\right)^{-1}-W(\ell) T_{\alpha} W(\ell)^{-1}\right\}
$$

the same as given in [7].

Similar to the situation in Sect. III, we can easily obtain the commutation relations for $\tilde{\delta}_{\alpha}^{(k)}$ 's $(k \in \mathbb{Z})$ by making use of the explicit expressions (52) and (55) (or (60)) for $\tilde{\delta}_{\alpha}^{(k)} W$. It turns out that

$$
\left[\tilde{\delta}_{\alpha}^{(m)}, \tilde{\delta}_{\beta}^{(n)}\right] W=\alpha^{a} \beta^{b} C_{a b}^{c} \tilde{\delta}_{c}^{(m+n)} W, \quad(m, n \in \mathbb{Z}) .
$$

This is another Kac-Moody algebra $G \otimes C\left[\ell, \ell^{-1}\right]$. But as we have warned before, $\tilde{\delta}_{\alpha}^{(k)} W$ with $k<0$ does not give a nontrivial symmetry transformation for the basic field $g(x)$ in the theory.

In ref. [7], the group structure for those "nontrivial" transformations was shown to be $G \otimes C\left[\ell, \ell^{-1}\right]$ too by working with their action on the configuration space of $g(x)$. However, as we will see in the next section, we can obtain the same result when working exclusively in the framework of the $\mathrm{RH}$ transform.

\section{General Group Theoretical Aspects of Infinitesimal RH Transforms}

Most parts of the discussion in the above two sections are actually quite general, independent of the 2-d principal chiral model. Both the expressions (e.g., Eqs. (21) and (50)) for infinitesimal RH transforms obtained there and the discussion of their Lie algebras (31) and (61) are generally true, provided that $U(\ell) \in G$ and $W(\ell) \in G$ analytic around $\ell=0$, and the kernal is formed according to Eqs. (6) and (39) with $u(\ell), \tilde{u}(\ell) \in G$. The special nonlinear model comes in only when the connection between $U(\ell)$ or $W(\ell)$ and the basic fields in the model is specified through the linearization equations supposed to be satisfied by $U(\ell)$ or $W(\ell)$. Further discussion of the group properties for transformations in nonlinear theories is better to be done exclusively within the framework of $\mathrm{RH}$ transforms, as the conclusions obtained in this way should be rather universal.

First let us discuss the commutators between the two infinite sets of RH

3 Note that in our previous approach in [7], we have $\delta_{\alpha}^{(0)} W(\ell)=0$, but in the present RH approach $\tilde{\delta}_{\alpha}^{(0)} W(\ell)$ is a global right-multiplication as given by Eq $(52)$ This is because the boundary condition we adopted for $W(\ell)$ in [7], i e $W(\ell ; x=\infty)=1$, is not respected by the RH transform. However, $\tilde{\delta}_{\alpha}^{(0)} g$ is the same in both approaches 
transforms $\left\{\delta_{a}^{(m)}\right\}$ and $\left\{\tilde{\delta}_{a}^{(m)}\right\}$. Since $W(\ell)$ satisfies the same linearization system as $U(\ell)$ does but with $\ell$ replaced by $1 / \ell$, so we can view $W(\ell)$ as the analytic continuation of $U(\ell)$ (this is allowable when the linearization equations for $U(\ell)$ do not have singularities at $\ell=0$ and $\ell=\infty$ ). With this continuation we can transfer any action on $W(\ell)$ to one on $U(\ell)$ and vice versa. Thus, we obtain

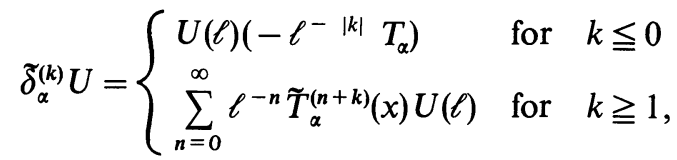

$$
\begin{aligned}
& \delta_{\alpha}^{(k)} W= \begin{cases}W(\ell)\left(-\ell^{-|k|} T_{\alpha}\right) & \text { for } \quad k<0, \\
-\sum_{n=1}^{\infty} \ell^{-n} T_{\alpha}^{(n+k)}(x) W(\ell) & \text { for } \quad k \geqq 0 .\end{cases}
\end{aligned}
$$

Also from Eqs. (30) and (60) we have

$$
\begin{aligned}
& \tilde{\delta}_{\alpha}^{\prime} U(\ell) \equiv \sum_{k=0}^{\infty} \ell^{\prime k} \tilde{\delta}_{\alpha}^{(k)} U(\ell)=\frac{\ell \ell^{\prime}}{1-\ell \ell^{\prime}}\left\{W\left(\ell^{\prime}\right) T_{\alpha} W\left(\ell^{\prime}\right)^{-1}-U(\ell) T_{\alpha} U(\ell)^{-1}\right\} \\
& \delta_{\alpha}^{\prime} W(\ell) \equiv \sum_{k=1}^{\infty} \ell^{\prime k} \delta_{\alpha}^{(k)} W(\ell)=\frac{1}{\ell \ell^{\prime}-1}\left\{U\left(\ell^{\prime}\right) T_{\alpha} U\left(\ell^{\prime}\right)^{-1}-W(\ell) T_{\alpha} W(\ell)^{-1}\right\}
\end{aligned}
$$

From these expressions one can see that $\tilde{\delta}_{\alpha}^{(k)} U(\ell),(k \geqq 1)$, and $\delta_{\alpha}^{(k)} W(\ell)(k \geqq 0)$ are really analytic around $\ell=0$.

Using these expressions, it is straightforward to compute the commutators. For example, we have

$$
\left[\delta_{\alpha}^{\prime}, \tilde{\delta}_{\beta}^{\prime \prime}\right] U=\alpha^{a} \beta^{b} C_{a b}^{c} \frac{1}{1-\ell^{\prime} \ell^{\prime \prime}}\left\{\ell^{\prime} \ell^{\prime \prime} \delta_{c}^{\prime} U+\tilde{\delta}_{c}^{\prime \prime} U\right\}
$$

Expanding both sides in powers of $\ell^{\prime}$ and $\ell^{\prime \prime}$, we obtain

$$
\begin{gathered}
\left\{\delta_{a}^{(0)}, \tilde{\delta}_{b}^{(m)}\right] U=C_{a b}^{c} \tilde{\delta}_{c}^{(m)} U, \\
{\left[\delta_{a}^{(n)}, \tilde{\delta}_{b}^{(m)}\right] U= \begin{cases}C_{a b}^{c} \delta_{c}^{(n-m)} U, & (m>1), \\
C_{a b}^{c} \delta_{c}^{(0)} U, & (n=m \geqq 1) \\
C_{a b}^{c} \tilde{\delta}_{c}^{(m-n)} U, & (m>n \geqq 1) .\end{cases} }
\end{gathered}
$$

Furthermore, ${ }^{4}$

$$
\begin{array}{ll}
{\left[\delta_{a}^{(-j)}, \tilde{\delta}_{b}^{(k)}\right] U=C_{a b}^{c} \tilde{\delta}_{c}^{(j+k)} U,} & (j, k \geqq 1), \\
{\left[\delta_{a}^{(j)}, \tilde{\delta}_{b}^{(-k)} U=C_{a b}^{c} \delta_{c}^{(j+k)} U,\right.} & (j, k \geqq 0) .
\end{array}
$$

It is very interesting that in the Lie algebra formed by $\delta_{a}^{(j)}$ and $\tilde{\delta}_{a}^{(k)}(j, k \in \mathbb{Z})$, there are two more Kac-Moody algebras, in addition to Eqs. (31) and (61): if we define

$$
P_{a}^{(m)}= \begin{cases}\delta_{a}^{(-m)}, & m>0, \\ \tilde{\delta}_{a}^{(m)}, & m \leqq 0,\end{cases}
$$

4 In evaluating these commutators one should keep in mind that both $\delta_{\alpha}^{(-j)} W$ and $\delta_{\alpha}^{(-j)} U(j \geqq 1)$ are not analytic around $\ell=0$ 
and

$$
Q_{a}^{(m)}= \begin{cases}\delta_{a}^{(m)}, & m \geqq 0, \\ \tilde{\delta}_{a}^{(-m)}, & m<0,\end{cases}
$$

then both of them form the Kac-Moody algebra $G \otimes C\left[\ell, \ell^{-1}\right]$ respectively:

$$
\left[P_{a}^{(m)}, P_{b}^{(m)}\right]=C_{a b}^{c} P_{c}^{(m+n)},\left[Q_{a}^{(m)}, Q_{b}^{(n)}\right]=C_{a b}^{c} Q_{c}^{(m+n)},(m, n \in \mathbb{Z}) .
$$

However, these two subalgebras do not commute. The RH transformations seem to have richer group-structure than the Kac-Moody algebra.

In this regard, it seems to me that the group structure of RH transforms depends on the homotopy of the closed loop $C$ on which the kernal is defined. We can examine the RH transforms acting on $U(\ell)$. The analytic properties (the singularities) of $U(\ell)$ are (at least partially) determined by the singularities of the linearization equations in $\ell$. The small circles $C$ in Sect III were chosen such that there are no singularities of $U(\ell)$ inside and for the circle $\widetilde{C}$ in Sect. IV there are no singularities of $U(\ell)$ outside. We have found that the two families of $\mathrm{RH}$ transformations with the kernal defined on $C$ and $\tilde{C}$ respectively are not the same and they do not commute.

In summary, generally speaking, RH transforms can form an infinitedimensional group. It has a Kac-Moody algebra $G \otimes C\left[\ell, \ell^{-1}\right]$ as its subalgebra, where $G$ is a finite dimensional group associated with the linearization system for the nonlinear theory under consideration, and may have richer structure. Because the RH transform method has been widely used in many nonlinear problems (see $[6,10$, $13,16]$ and references in [14]), on the grounds of the generality of our present discussion we can make the assertion that at least one Kac-Moody algebra will appear in those nonlinear models which can be solved by the RH transform method, although the details for the hidden symmetry transformations for the basic fields in each case has to be worked out individually. Especially, we would like to point out that our discussion in this paper can be applied to 4-d self-dual Yang-Mills systems, as the so-called $J$-formulation of it is formally very similar to 2 -d principal chiral models.

Finally, the results of this paper also suggest that we can use the finite RH transformations to exponentiate the infinitesimal hidden symmetry transformations proposed by us previously (see, e.g., [7] and [12]), as Hauser, Ernst did for the infinitesimal Kinnersley-Chitre transformations [17] in the case of axial symmetric vacuum gravity.

Acknowledgements. It is my pleasure to thank Prof. S. Adler and the Institute for Advanced Study Prof. W. Zimmermann and the Max-Planck Institute for Physics and Astrophysics for the warm hospitality I received during my visit when this work began and continued. Especially, I am very grateful to Prof. J. M. Souriau and A. Rouet and the Center for Theoretical Physics at Marseille for the warm hospitality extended to me. I would like to thank Dr B. Julia for interesting discussions.

Note added. After submitting this paper I learned that F. A. Bais and R. Sasaki have reached similar conclusions based on their previous work, "On the complete integrability of the static axially symmetric self-dual gauge field equations for an arbitrary group," Nucl. Phys. B195 (1982) 522 and "Solution generation techniques for static axially symmetric self-dual gauge field equations," Phys. Lett. 113B (1982) 
35 and "Algebraic construction of static axially symmetric self-dual gauge fields for an arbitrary group," Phys. Lett. 113B (1982) 39

\section{References}

1 Dolan, L, Roos A.: Nonlocal currents as Noether currents. Phys Rev. D22, 2018 (1980)

2 Hou, B. Y.: Yale preprint 80-29, 1980 (unpublished); Hou, B. Y., Ge, M. L., Wu, Y. S. : Noether analysis for the hidden symmetry responsible for an infinite set of nonlocal currents Phys. Rev D24, $2238(1981)$

3 Dolan, L.: Kac-Moody algebra is hidden symmetry of chiral models. Phys. Rev. Lett. 47, 1371 (1981)

4. Ge, M L., Wu, Y. S.: An explicit approach to the group structure of hidden symmetry Phys. Lett. 108B, 411 (1982)

5 Devchand, C, Fairlie, D B.: A generating function for hidden symmetries of chiral models Nucl. Phys B194, 232 (1982)

6 Ueno, K : RIMS preprint 374, 1981

7 Wu, Y S.: Extension of the Hidden Symmetry algebra in classical chiral models. Nucl Phys. B211, $160(1983)$

8 Forgacs, P, Harvath, Z, Palla, L : Towards complete integrability of the self-dual equations Phys Rev D23, 1876 (1981)

9 Chau, L L, Ge, M L, Wu, Y S : Noether currents and algebraic structure of the hidden symmetry for super chiral fields Phys Rev D25, 1080(1982); Kac-Moody algebra in the self-dual Yang-Mills equation. Phys. Rev D25, 1086 (1982)

10. Ueno, K., Nakamura, $Y$ : Transformation theory for anti-dual equations and the Riemann-Hilbert problem Phys Lett 109B, 273 (1982)

11 Chau, L. L., Wu, Y S.: More about hidden-symmetry algebra for the self-dual Yang-Mills system. Phys. Rev D26, 3581 (1982)

12. Chau, L. L, Ge, M L., Sinha, A, Wu, Y S : Hidden symmetry algebra for the self-dual Yang-Mills equation. Phys Lett, 121B, 391 (1983)

13 Zakharov, V. E., Shabat, A. B.: Funct. Anal. Appl. 13, 13 (1979)

14. For a brief review see, e.g., Mikhailov, A. V : CERN preprint TH-3194, 1981, and references therein

15 Kac, V G : Math USSR-IZV. 2, 1311 (1968); Moody, R V : J Algebra 10, 211 (1968)

16 Hauser, I., Ernst, F J : Integral equation method for effecting Kinnerstey-Chitre transformations and-II. Phys Rev., D20, 362 and 978 (1979)

17 Kinnersley, W., Chitre, D M : "Symmetries of the stationary Einstein-Maxwell field equations II," and "-III" J Math Phys 18, 1538 (1977) and 19, 1926 (1978)

Communicated by $\mathbf{R}$ Stora

Received September 16, 1982; in revised form November 23, 1982 